\title{
Enhanced ROS-Generation in Lymphocytes from Alzheimer's Patients
}

\author{
S. Leutner ${ }^{1}$ \\ K. Schindowski ${ }^{1}$ \\ L. Frölich ${ }^{2,3}$ \\ K. Maurer ${ }^{2}$ \\ T. Kratzsch ${ }^{2}$ \\ A. Eckert ${ }^{1,4}$ \\ W. E. Müller ${ }^{1}$
}

Introduction: Reactive oxygen species (ROS) have been implicated in neurodegeneration and seem to be involved in the physiology and pathophysiology of several diseases, including normal aging and Alzheimer's disease (AD). Enhanced ROS production in aging or $A D$ is not restricted to the brain, but can also been seen in several peripheral tissues. The objective of the present study was to evaluate whether the mechanisms involved in the generation of oxidative stress in normal senescence and Alzheimer's disease are identical or not. Methods: We analysed intracellular basal levels of ROS in lymphocytes from AD patients and healthy young and aged not-demented subjects as well as ROS levels following stimulation with d-ribose and staurosporine in all three groups. ROS levels were measured by flow cytometry using the intracellular fluorescence dye dihydrorhodamine 123 (DHR123). Results: Our study shows that AD lymphocytes have increased basal levels of ROS, low susceptibility to ROS stimulation by 2-deoxy-D-ribose (dRib) and an increased response to staurosporine when compared with age-matched controls. Discussion: The data suggest that the defect(s) responsible for enhanced ROS production in AD may involve different or additional biological pathways than those involved in enhanced ROS generation during aging.

\section{Introduction}

Several hypotheses have been proposed as molecular basis explaining the enhanced neurodegeneration occurring during normal aging and Alzheimer's disease (AD). One of the most compelling is the role of free radical-induced oxidative stress in these disorders $[1,16,17,22,24,25]$. Enhanced oxidative stress during aging and $\mathrm{AD}$ is not restricted to the brain but also present in peripheral cells like fibroblasts [11] or lymphocytes $[6,22,23]$. Several oxidative stress-related changes in lymphocytes have been observed related to aging as for example diminished concentrations of antioxidants like $\alpha$-tocopherol, ascorbat and glutathione [15] or age-associated changes in mitochondrial function including a decrease of several complexes of the respiratory chain and increased levels of oxidized mitochondrial DNA $[6,12]$.

Some of these changes ave additionally elevated in peripheral cells from patients with Alzheimer's disease (AD) like decreased glutathione (GSH) content [3] increased levels superoxide dismutase (MnSOD) mRNA [5] or increased lipid peroxidation [4]. Some findings indicate increased oxidative damage in lymphocytes of Alzheimer's disease patients [18-21]. Moreover, similar changes may be induced by AD specific mutations of APP and PS1 genes. Gibson et al. demonstrated that fibroblasts bearing the AD Presenilin-1 246 Ala $\rightarrow$ Glu mutation have altered means of hand-

Affiliation

${ }^{1}$ Department of Pharmacology, Biocenter, J. W. Goethe University of Frankfurt, Marie-Curie-Str. 9, 60439 Frankfurt am Main, Germany

2 Department of Psychiatry and Psychotherapy I, J. W. Goethe University of Frankfurt, Heinrich-Hoffmann-Str. 10, 60528 Frankfurt am Main, Germany

${ }^{3}$ Present Address: Central Institute of Mental Health, J5, 68159 Mannheim, Germany

${ }^{4}$ Present Address: Neurobiology Research Laboratory, Psychiatric Clinic, University of Basel, Wilhelm Klein-Str. 27, 4025 Basel, Switzerland

Correspondence

Walter E. Müller, Ph. D. · Department of Pharmacology · J. W. Goethe-University · Biocenter · Niederursel · Marie-Curie-Str. 9 • 60439 Frankfurt · Phone: +49 6979829373 · Fax: +496979829374 .

E-Mail: pharmacolnat@em.uni-frankfurt.de

Received 1.5.2005 • Revised 1.7.2005 • Accepted 8.7.2005

Bibliography

Pharmacopsychiatry 2005; 38: 312-315 - ( ) Georg Thieme Verlag KG Stuttgart · New York DOI 10.1055/s-2005-916186

ISSN 0176-3679 
ling oxidative stress [10]. Comparable findings were reported for lymphocytes of mice bearing PS1 mutations [9].

In order to investigate whether similar or divergent mechanisms of ROS generation are relevant for aging and sporadic AD, we investigated levels of ROS in young and aged human lymphocytes as well as in lymphocytes from AD-patients. In contrast to measurements of plasma lipid peroxidation products or the activities of antioxidant enzymes in blood cells, determination of intracellular ROS (reactive oxygen species) may directly reflect oxidative stress inside the cell.

\section{Materials and Methods}

\section{Subjects}

For the study comparing ROS levels in young and aged humans, we used fresh blood samples from $n=47$ healthy subjects (21 women and 26 men) between 20 and 86 years. There were 10 women and 13 men in the aged group $(n=23$; aged over 60 years, mean age $69.4 \pm 6.8$ years) and 11 women and 13 men in the group of young controls ( $n=24$; age under 35 years, mean age $27.38 \pm 3.81$ years).

For the determination of ROS levels in lymphocytes from aged not-demented controls and patients with Alzheimer's disease, blood samples were taken from $n=53$ subjects ( 33 women and 20 men) between 57 and 91 years. There were 14 women and 11 men in the aged group ( $n=25 ; 60-85$ years, mean age $71.7 \pm 7.5$ years) and 19 women and 9 men in the Alzheimer's group ( $n=28$; 57-91 years, mean age $72.7 \pm 10.0$ years). Dementia was diagnosed according to ICD-10. Diagnosis of Alzheimer's disease was achieved following the guidelines of the National Institute of Neurological and Communicative Disorders and StrokeAlzheimer's Disease and Related Disorders (NINCDS-ADRDA criteria) task force. The majority of cases exhibited mild to moderate dementia.

Subjects with pathological distribution of lymphocyte subpopulations in flow-cytometric analysis were rejected from the study. Most of the patients were taking drugs against cardiovascular disorders. None of the control patients received psychotropic medication, drugs with known effects on the immune system, or antioxidative drugs. The study was approved by the responsible Ethical Committee and written informed consent from all subjects was received, where appropriate, from their caregivers.

\section{Cells}

Peripheral blood lymphocytes were prepared from whole fresh blood by separating on Ficoll (Biochrom KG, Berlin, Germany) as previously described [8]. Cells were investigated after the incubation with two different stimuli or with buffer as control.

\section{Measurement of oxidative stress}

The determination of ROS was performed according to a previously described procedure [22]. In brief, lymphocytes were incubated with the dye dihydrorhodamine123 (DHR123; $10 \mu \mathrm{mol} /$ 1; Molecular Probes, Netherlands) for 15 minutes at $37^{\circ} \mathrm{C}$. Then, the conversion of DHR123 to its fluorescent derivative rhodamine123 was detected by FACS analysis. Probes were stimulated either for 16 hours with $50 \mathrm{mM}$ 2-deoxy-D-ribose (dRib) or 500 nM staurosporine.

\section{Results}

\section{Altered generation of ROS in aging}

Oxidative stress was induced with either 2-deoxy-D-ribose (dRib) or the apoptotic agent staurosporine. Cells were incubated for 16 hours at $37^{\circ} \mathrm{C}$ after the addition of one of the stimuli mentioned or of buffer as control. The oxidative-sensitive fluorescent dye DHR123 was used to detect presence of ROS in human lymphocytes. Basal levels of ROS (expressed as mean fluorescence intensity in arbitrary units) accumulated significantly in aged human lymphocytes (Fig. 1A; ${ }^{*} p<0.05 ; n=22-24$ ).

Incubation with 2-deoxy-D-ribose (dRib), a reducing sugar that induces apoptosis in quiescent human lymphocytes through oxidative mechanisms [13], led to an age-related increase in ROS levels (Fig. 1B; ${ }^{*} p<0.05 ; n=21-23$ ). In addition, treatment with the apoptotic stimuli staurosporine generates increased levels of ROS without any age-related differences (Fig. 1C; $n=16$ ).

\section{Altered generation of ROS in Alzheimer's disease}

After a period of 16 hours in vitro incubation, basal levels of ROS were significantly increased in lymphocytes of Alzheimer's patients (Fig. 2A; ${ }^{* *} \mathrm{p}<0.01 ; n=23-25$ ). The incubation with dRib (50 mM) led to an increase in DHR123 fluorescence without any Alzheimer-related difference (Fig. 2B; $n=24-28$ ).

In contrast, treatment with staurosporine ( $500 \mathrm{nM}$ ) evoked a significantly larger increase in ROS levels in lymphocytes from Alzheimer's patients than from healthy controls (Fig. 2C; ${ }^{*} \mathrm{p}<0.05$; $n=23-25)$.

\section{Discussion}

Only few observations indicating enhanced oxidative stress have been reported for lymphocytes from AD patients, such as de-

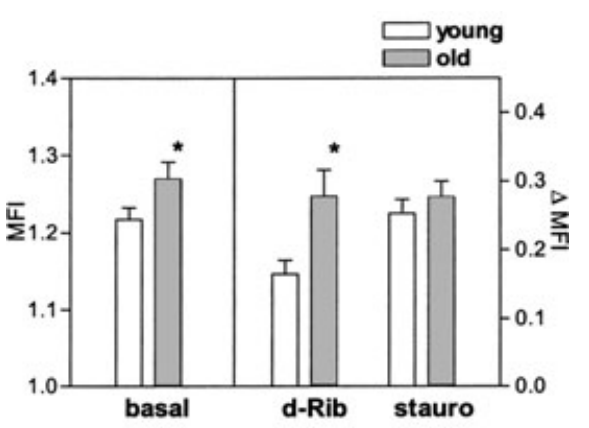

Fig. 1 Levels of ROS in lymphocytes from young and old subjects measured with the fluorescent dye DHR123. A) Basal levels of ROS in human lymphocytes. Significantly higher levels could be determined in aging (old, $n=22$ versus young, $n=$ 24; * ${ }^{*}$ <.05). B) Induced ROS levels over control in lymphocytes cultured for $16 \mathrm{~h}$ with $\mathrm{dRib}$. There was an age-related increase in DHR123 fluorescence ( $\Delta$ increase over baseline; old, $n=23$; versus young, $\left.n=21 ;{ }^{*} p<0.05\right)$. C) Induced ROS levels over control in lymphocytes cultured for $16 \mathrm{~h}$ with staurosporine. There was an increase in ROS without any age-related differences ( $\Delta$ increase over baseline; old, $n=16$; versus young, $n=16$ ). 


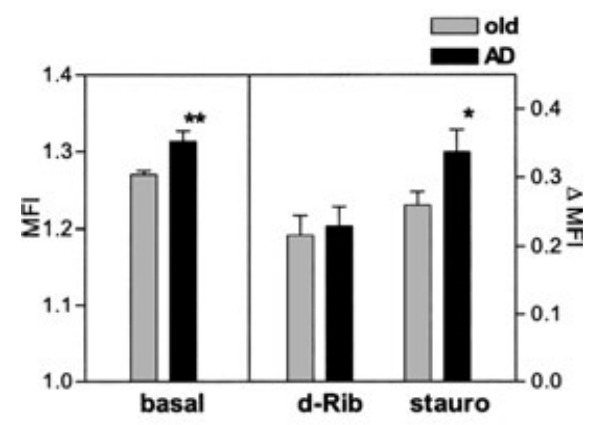

Fig. 2 Levels of ROS in lymphocytes from old subjects and patients with Alzheimer's disease (AD) measured with the fluorescent dye DHR123. A) Basal levels of ROS in human lymphocytes. Significantly higher levels could be determined in $A D(A D$, $n=23$; versus old, $\left.n=25 ;{ }^{*} p<0.01\right)$. B) Induced ROS levels over control in lymphocytes cultured for $16 \mathrm{~h}$ with dRib. There was an increase in DHR123 fluorescence without any age-related differences ( $\Delta$ increase over baseline; AD, $n=28$; versus old, $n=24$ ). C) Induced ROS levels over control in lymphocytes cultured for $16 \mathrm{~h}$ with staurosporine. There was an age-related increase in ROS ( $\Delta$ increase over baseline; $\mathrm{AD}, n=23$; versus old, $n=25$; $\left.^{*} p<0.05\right)$.

creased glutathione (GSH) content [3], altered calcium dynamics [7] as well as enhanced oxidative DNA damage [18]. The experiments in the current study demonstrate that cells from AD patients handle oxidative stress differently than aged-matched controls when incubated with two different stimuli of ROS.

The reducing sugar 2-deoxy-D-ribose (dRib) has been shown to induce apoptosis in a variety of cells. Apoptosis seems to be generated by free radicals and mitochondrial dysfunction. A timedependent marked increase of depolarised mitochondria was observed in cultures exposed to dRib, possibly occurring as a consequence of both, production of radicals and/or significant glutathione depletion. We have already previously shown that treatment with dRib evokes increased levels of ROS in human lymphocytes [22]. Other authors report that dRib treatment induced complete depletion of reduced glutathione (GSH), a key molecule for anti-oxidant protection [13]. They hypothesize that dRib, by lowering the intracellular levels of GSH until complete depletion leads to an oxidative imbalance inside cells. There are also findings indicating disturbances of cytoskeletal integrity and cell adhesion [13].

Staurosporine is widely employed as an inducer of apoptosis in many cell types. The intracellular cascades that mediate staurosporine-induced apoptosis are largely unknown. ROS have been implicated in staurosporine-induced changes of mitochondrial membrane potential, cell cycle arrest and the activation of caspase-3 by the accumulation of cytochrome $\mathrm{c}$ in the Cytosol [2]. It has been shown that mitochondrial respiratory chain is the principal source of ROS generation in staurosporine-induced apoptosis [2]. Moreover, staurosporine induces an early increase in cytosolic calcium followed by delayed increase of mitochondrial calcium. Mitochondrial ROS accumulation is also described [14].

In our study, basal ROS levels were increased in aged subjects and were further increased in lymphocytes from $A D$ patients. These results confirm the above mentioned observations indicating additionally altered metabolism of free radicals also in cells from $\mathrm{AD}$ patients.
Stimulation of lymphocytes from young and old donors showed no age-related difference in the extent of ROS-generation when incubated with staurosporine, however, treatment with $\mathrm{dRib}$ evokes significant higher ROS generation in cells from aged subjects than in young controls. Since $\mathrm{dRib}$ is particularly effective in depleting intracellular levels of GSH, the already decreased intracellular levels of glutathione in lymphocytes from aged relative to young subjects may be an explanation for the latter findings [15].

Lymphocytes from AD patients show no differences in ROS generation compared to age-matched controls when incubated with dRib, suggesting that levels of glutathione are not further decreased in cells from AD patients. On the other hand, cells from AD patients but not from aged humans are more sensitive to ROS generation induced by staurosporine. The exact mechanism remains unknown. We can only speculate that the above mentioned alterations of mitochondrial function in AD lymphocytes, might contribute to the enhanced sensitivity to the staurosporine [23-25].

The main finding of the present study is that two different stimuli which trigger the generation of free radicals in human lymphocytes via different mechanisms evoke different answers in cells from aged subjects and patients with Alzheimer's disease. We can conclude that the mechanisms involved in the generation of free radicals are different in the pathology of Alzheimer's disease and in senescence.

\section{References}

${ }^{1}$ Butterfield DA, Howard B, Yatin S, Koppal T, Drake J, Hensley K, Aksenov M, Aksenova M, Subramaniam R, Varadarajan S, Harris-White ME, Pedigo NWJ, Carney JM. Elevated oxidative stress in models of normal brain aging and Alzheimer's disease. Life Sci 1999; 65: 1883-1892

${ }^{2}$ Cai J, Wallace DC, Zhivotovsky B, Jones DP. Separation of cytochrome c-dependent caspase activation from thiol-disulfide redox change in cells lacking mitochondrial DNA. Free Radic Biol Med 2000; 29: $334-342$

${ }^{3}$ Cecchi C, Latorraca S, Sorbi S, Iantomasi T, Favilli F, Vincenzini MT, Liguri G. Gluthatione level is altered in lymphoblasts from patients with familial Alzheimer's disease. Neurosci Lett 1999; 275: 152 - 154

${ }^{4}$ Cecchi C, Fiorillo C, Sorbi S, Latorrace S, Nacmias B, Bagnoli S, Nassi P, Liguri G. Oxidative stress and reduced antioxidant defenses in peripheral cells from familial Alzheimer's patients. Free Radic Biol Med 2002; 33: $1372-1379$

${ }^{5}$ De Leo ME, Borrello S, Passantino M, Palazzotti B, Mordente A, Daniele A, Filippini V, Galeotti T, Masullo C. Oxidative stress and overexpression of manganese superoxide dismutase in patients with Alzheimer's disease. Neurosci Lett 1998; 250: $173-176$

${ }^{6}$ Drouet M, Lauthier F, Charmes JP, Sauvage P, Ratinaud MH. Age-associated changes in mitochondrial parameters on peripheral human lymphocytes. Exp Gerontol 1999; 34: 843-852

${ }^{7}$ Eckert A, Forstl H, Zerfass R, Oster M, Hennerici M, Muller WE. Changes of intracellular calcium regulation in Alzheimer's disease and vascular dementia. J Neural Transm Suppl 1998; 54: 201 -210

${ }^{8}$ Eckert A, Hartmann H, Muller WE. Beta-Amyloid protein enhances the mitogen-induced calcium response in circulating human lymphocytes. FEBS Lett 1993; 330: 49-52

${ }^{9}$ Eckert A, Schindowski K, Leutner S, Luckhaus C, Touchet N, Czech C, Müller WE. Alzheimer's disease-like alterations in peripheral cells from Presenilin-1 transgenic mice. Neurobiol Disease 2001; 8: 331 342

${ }^{10}$ Gibson GE, Zhang H, Sheu KR, Park LC. Differential alterations in antioxidant capacity in cells from alzheimer patients. Biochim Biophys Acta 2000; 15: 319-329 
${ }^{11}$ Huang HM, Fowler C, Xu H, Zhang H, Gibson GE. Mitochondrial function in fibroblasts with aging in culture and/or Alzheimer's disease. Neurobiol Aging 2005; 26: 839-848

${ }^{12}$ King CM, Gillespie ES, McKenna PG, Barnett YA. An investigation of mutation as a function of age in humans. Mutat Res 1994; 316: 79-90

${ }^{13}$ Kletsas D, Barbieri D, Stathakos D, Botti B, Bergamini S, Tomasi A, Monti D, Malorni W, Franceschi C. The highly reducing sugar 2-deoxy-Dribose induces apoptosis in human fibroblasts by reduced glutathione depletion and cytoskeletal disruption. Biochem Biophys Res Commun 1998; 243: $416-425$

${ }^{14}$ Kruman II, Mattson MP. Pivotal role of mitochondrial calcium uptake in neural cell apoptosis and necrosis. J Neurochem 1999; 72: $529-$ 540

${ }^{15}$ Lenton KJ, Therriault H, Cantin AM, Fulop T, Payette H, Wagner JR. Direct correlation of glutathione and ascorbate and their dependence on age and season in human lymphocytes. Am J Hum Genet 2000; 71: $1194-1200$

${ }^{16}$ Leutner S, Eckert A, Müller WE. ROS generation, lipid peroxidation and antioxidant enzyme activites in the aging brain. J Neural Transm 2001; 108: 955-967

${ }^{17}$ Markesbery WR. Oxidative stress hypothesis in Alzheimer's disease. Free Radic Biol Med 1997; 23: 134- 147

${ }^{18}$ Mecocci P, Polidori MC, Ingegni T, Cherubini A, Chionne F, Cecchetti R, Senin U. Oxidative damage to DNA in lymphocytes from AD patients. Neurology 1998; 51: 1014-1017
${ }^{19}$ Mecocci P, polidori C, Cherubini A, Ingegni T, Mattioli P, Catani M, Rinaldi P, Ceccetti R, Stahl W, Senin U, Beal F. Lymphocyte oxidative DNA damage and plasma antioxidants in Alzheimer disease. Arch Neurol 2002; 59: $794-798$

${ }^{20}$ Migliore L, Fontana I, Trippi F, Colognato R, Coppedé F. Tognoni G, Nucciarone B, Siciliano G. Oxidative DNA damage in peripheral leukocytes of mild cognitive impairment and AD patients. Neurobiol Aging 2005; 26: $567-573$

${ }^{21}$ Morocz M, Kalman J, Juhasz A, Sinko I, McGlynn AP, Downes CS, Janka Z, Rasko I. Elevated levels of oxidative DNA damage in lymphocytes from patients with Alzheimer's disease. Neurobiol Aging 2002; 23: $47-53$

22 Schindowski K, Leutner S, Muller WE, Eckert A. Age-related changes of apoptotic cell death in human lymphocytes. Neurobiol Aging 2000; 21: $661-670$

${ }^{23}$ Schindowski K, Kratsch T, Peters J, Steiner B, Leutner S, Touchet N, Maurer K, Czech C, Pradier L, Frölich L, Müller WE, Eckert A. Impact of aging, sporadic and genetic risk factors on vulnerability to apoptosis in Alzheimer's disease. Neuromolecular Med 2003; 4: 161 - 177

${ }^{24}$ Smith MA, Rottkamp CA, Nunomura A, Raina AK, Perry B. Oxidative stress in Alzheimer's disease. Biochim Biophys Acta 2000; 1502: $139-144$

${ }^{25}$ Smith MA, Nunomura A, Lee H, Zhu X, Moreira PI, Avila J, Perry G. Chronological primacy of oxidative stress in Alzheimer disease. Neurobiol Aging 2005; 26: 579-588 